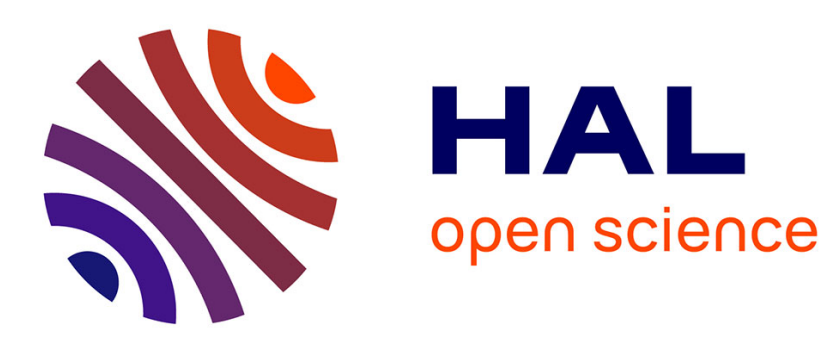

\title{
A Novel Hierarchical Low Complexity Synchronization Method for OFDM Systems
}

Pramod Udupa, Olivier Sentieys, Pascal Scalart

\section{To cite this version:}

Pramod Udupa, Olivier Sentieys, Pascal Scalart. A Novel Hierarchical Low Complexity Synchronization Method for OFDM Systems. 2013 IEEE 77th Vehicular Technology Conference (VTC Spring), Jun 2013, Dresden, Germany. pp.1-5, 10.1109/VTCSpring.2013.6691838 • hal-00931530

\section{HAL Id: hal-00931530 \\ https://hal.inria.fr/hal-00931530}

Submitted on 15 Jan 2014

HAL is a multi-disciplinary open access archive for the deposit and dissemination of scientific research documents, whether they are published or not. The documents may come from teaching and research institutions in France or abroad, or from public or private research centers.
L'archive ouverte pluridisciplinaire HAL, est destinée au dépôt et à la diffusion de documents scientifiques de niveau recherche, publiés ou non, émanant des établissements d'enseignement et de recherche français ou étrangers, des laboratoires publics ou privés. 


\title{
A Novel Hierarchical Low Complexity Synchronization Method for OFDM Systems
}

\author{
Pramod Udupa, Olivier Sentieys, and Pascal Scalart \\ IRISA, University of Rennes 1, France \\ Email:\{pudupa, sentieys, pascal.scalart\}@irisa.fr
}

\begin{abstract}
A new hierarchical synchronization method is proposed for initial timing synchronization in orthogonal frequencydivision multiplexing (OFDM) systems. Based on the proposal of new training symbol, a threshold based timing metric is designed for accurate estimation of start of OFDM symbol in a frequency selective channel. Threshold is defined in terms of noise distributions and false alarm, which makes it independent of type of channel it is applied. Frequency offset estimation is also done for the proposed training symbol. The performance of the proposed timing metric is evaluated using simulation results. The proposed method achieves low mean squared error (MSE) in timing offset estimation at five $(5 \mathbf{x})$ times lower computational complexity compared to cross-correlation based method in a frequency selective channel. It is also computationally efficient compared to hybrid approaches for OFDM timing synchronization.
\end{abstract}

\section{INTRODUCTION}

OFDM systems are sensitive to timing and frequency synchronization [1] errors. Loss of timing synchronization causes inter-carrier interference (ICI) and inter-symbol interference (ISI). Also, frequency offset causes loss of orthogonality among sub-carriers which leads to ICI. These synchronization errors, if left uncompensated, reduces the advantage provided by OFDM systems over single-carrier systems.

Many methods have been proposed for initial timing synchronization previously. The synchronization methods have either been based on cyclic prefix or known preamble symbols [2] [3] [4] [5] [6]. Cyclic prefix (CP) based methods utilize the cyclic prefix length for synchronization which constitutes only a small part of the OFDM symbol. In a multipath channel, cyclic prefix samples are corrupted due to ISI. This leads to reduction in the number of uncorrupted samples for synchronization which leads to reduction in the accuracy of estimation.

Preamble-based synchronization methods utilize specially designed training symbol for achieving synchronization. Preamble symbols are designed with specific structure for maximizing the detection of start point of the OFDM symbol. Schmidl and Cox [2] proposed a training symbol (TS) which consists of two repetitive parts. The correlation peak of the timing metric has a plateau equal to the length of cyclic prefix in AWGN channel. The length of plateau is equal to length of the uncorrupted portion of the cyclic prefix in frequency selective (ISI) channels. The training symbol also allows a frequency estimation of one subcarrier spacing. Minn et al. [3] proposed an algorithm consisting of multiple repetitive parts with specific sign pattern for timing synchronization. The algorithm has steeper timing roll-off compared to Schmidl's timing metric roll-off. Frequency estimation was done using algorithm by Morelli [7]. The symbol has a frequency estimation range of $\pm L / 2$ sub-carrier spacings, where $L$ is the number of repetitive parts in the TS. Shi and Serpedin [4] proposed a four part TS which is similar to Minn's, but the algorithm used for timing estimation is more generalized and uses all repetitive parts of the TS. The frequency estimation range is \pm 2 sub-carrier spacing.

Park et al. [5] proposed a new training symbol which utilizes conjugate symmetry property for timing synchronization. The timing metric has a steeper roll-off compared to Minn's method. Choi [6] proposed a training symbol for multipath channels which also utilizes conjugate symmetry for correlation operation. The symbol is based on CAZAC (Zadoff-Chu) sequence, which has constant amplitude and has much better correlation properties than m-sequences. The timing metric has impulse like roll-off at the correct starting point. Although these methods [6], [5] have very steep timing roll-off metric, they are computationally intensive in number of operations required per point of timing metric.

Zhou [8] proposed a hybrid method for synchronization using preamble symbols. The method uses both kinds of correlation (auto and cross correlation) operations with TS and multiple thresholds for detection of first path in multipath channel. But it uses both correlation operations together simultaneously, thus using a lot of computations per timing metric point calculation. This leads to low throughput in output timing metric computation and hence can cause delay in synchronization.

In this paper, a novel low complexity hierarchical synchronization method is proposed. A new training symbol which has both repeating patterns and conjugate symmetry property is used. The training symbol is based on modified Chu (CAZAC) [9] sequence, which has smaller alphabet size than Zadoff-Chu sequences. The hierarchical timing metric proposed uses delay correlation in the first step and conjugate symmetry correlation in the second step. The first step is computationally efficient and operates on larger number of samples, while second step is computationally expensive but operates on fixed number of samples. The hierarchical method gains complexity advantage and good MSE performance by the auto-correlation (first step) and cross-correlation (second step) respectively.

The paper is arranged as follows. In Section II, the sys- 
tem model used for the timing synchronization method is described. Sections III and IV explain the new proposal for timing and frequency synchronization. In Section V, results of new proposal are compared to previous methods. Finally, Section VI concludes the paper.

\section{OFDM SIGNAL DESCRIPTION}

The transmitted baseband OFDM samples can be written in terms of IFFT equation as

$$
x[n]=\frac{1}{\sqrt{N}} \sum_{k=0}^{N-1} X[k] \cdot e^{j 2 \pi n k / N}
$$

where $N$ is the number of sub-carriers and $X_{k}$ the complex information carrying symbol in frequency domain. The sampled signal at the receiver can be written as

$$
r[n]=s[n-\eta] \cdot e^{j(2 \pi \epsilon n / N+\phi)}+w[n]
$$

where $\eta$ is the integer timing offset, $\epsilon$ the frequency offset and $\phi$ the phase offset. $w[n]$ is the additive white Gaussian noise (AWGN) and $s[n]$ in multipath channel is given by

$$
s[n]=\sum_{m=0}^{L_{h}-1} h[m] \cdot x\left[n-\tau_{m}\right]
$$

where $h$ is the sampled channel response (complex channel coefficients) at the receiver. $L_{h}$ is the number of channel paths and $\tau_{m}$ is the path delay corresponding $\mathrm{m}^{\text {th }}$ channel path. The channel is assumed static for the duration of the OFDM symbol.

\section{Proposed Timing Synchronization}

Timing synchronization is the step of finding the starting of the OFDM symbol. Frame and Symbol synchronization follow directly from timing synchronization in OFDM. Timing synchronization involves finding the unknown time offset $(\eta)$ in the presence of other unknowns $(\epsilon$ and $\phi)$. Since timing synchronization algorithms operate in presence of these other offsets, they have to be immune to them. Many algorithms have been proposed for timing synchronization which either utilize built-in structure, namely cyclic prefix or use a preamble which has a known structure [2], [3]. Preamble-based algorithms are more suitable for initial synchronization and have more range in frequency offset estimation compared to cyclic prefix based methods.

To achieve synchronization in ISI channel at low complexity, a new synchronization method is proposed based on proposal of a new training symbol. The training symbol has low PAPR and can support both delay correlation and conjugate symmetry correlation operations. The training symbol is generated using CAZAC sequence, which have very low PAPR and possess impulse-like auto-correlation properties and constant cross-correlation property.

The training sequence is based on modified Chu (CAZAC) [9] sequence, which have smaller alphabet size than Chu sequence. The modified Chu sequence [9] is given by

$$
a_{k}^{(r)}= \begin{cases}\exp \left(i \frac{2 \pi}{N_{s}}\left\lfloor\frac{r k^{2}}{2}\right\rfloor\right), & \text { for } N_{s} \text { even } \\ \exp \left(i \frac{2 r \pi k(k+1)}{N_{s}}\right), & \text { for } N_{s} \text { odd }\end{cases}
$$

where $0 \leq k<N_{s}, \operatorname{gcd}\left(r, N_{s}\right)=1$ and $\lfloor a\rfloor$ denotes the integer part of $a$. Here $r=1$ is used. The alphabet size is $N_{s}$ for modified Chu sequence compared to $2 N_{s}$ for Chu sequence.

The training symbol proposed is

$$
[C C C-C], C=\left[\begin{array}{ll}
A & B
\end{array}\right], B=A^{*}[-n]
$$

The part $\boldsymbol{A}$ is constructed by taking IFFT of the generated modified Chu sequence of size $N_{s}=\frac{N}{8}$. Then $\boldsymbol{B}$ is constructed from $\boldsymbol{A}$ by time-reversal and conjugation operation. The sign pattern $[+1+1+1-1]$ is designed to ensure steep roll-off for initial estimation algorithm. The length of a single part is designed to be greater than maximum delay spread of the multipath channel. The initial timing metric proposed for the training symbol is delay based auto-correlation method that involve using repeating pattern for delay based correlation. The timing metric for coarse initial estimate is:

$$
M_{\text {init }}[n]=\left(\frac{L}{L-1} \cdot \frac{|P[n]|}{R[n]}\right)^{2}
$$

where $P[n]$ is the auto-correlation function, $R[n]$ is the energy of the sequence and $L$ is the number of parts (here $L=4$ ) in the training symbol. The term $L /(L-1)$ is used to normalized for maximum value of 1 at the correct starting point. The expressions for $P[n]$ and $R[n]$ are

$$
\begin{aligned}
& P[n]=\sum_{k=0}^{L-2} u[k] \sum_{m=0}^{M-1} r^{*}[n+k M+m] \cdot r[n+(k+1) M+m] \\
& R[n]=\sum_{k=0}^{L-1} \sum_{m=0}^{M-1}|r[n+k M+m]|^{2}
\end{aligned}
$$

where $u[k]=p[k] \cdot p[k+1], p[k]$ contains the sign pattern of $[+1+1+1-1], k=0,1, \ldots,(L-1)$ and $M=N / L$. The time index corresponding to the maximum value gives the initial estimate.

$$
\widehat{\eta}_{\text {init }}=\arg \max _{n} M_{\text {init }}[n]
$$

Figure 1a shows the plot of $M_{i n i t}[n]$ for a Signal-to-Noise Ratio (SNR) of $20 \mathrm{~dB}$ in a frequency selective channel. The maximum peak is not exactly at zero which is the actual start of the OFDM symbol, but slightly shifted to the right due to multipath effect. The fine estimation algorithm consists of correcting this unknown shift and finding the correct starting point. The fine estimation algorithm does not assume a dominant first path and can work with non-dominant first path in multipath channel.

The fine estimation algorithm uses the conjugate symmetry present in the training symbol to estimate the correct starting point. The fine time estimation algorithms starts from the 

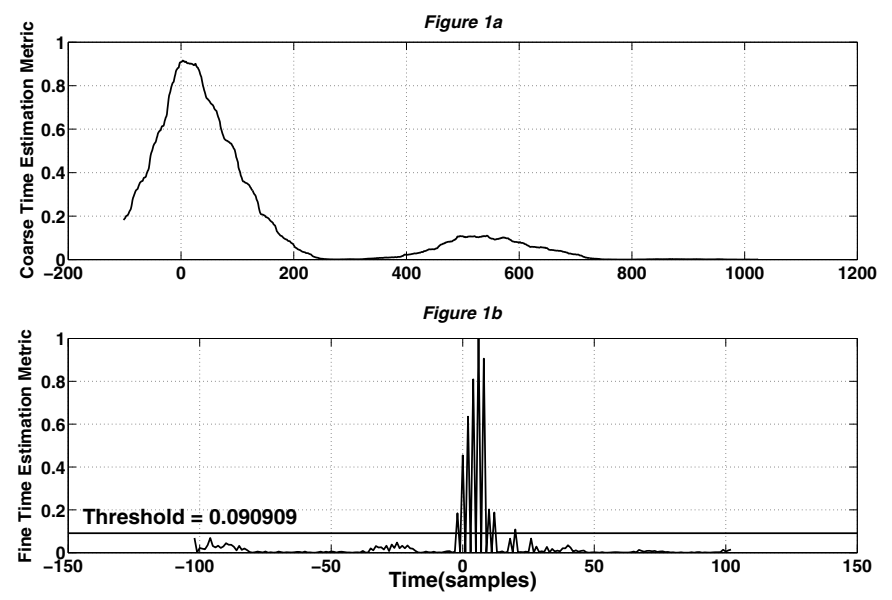

Fig. 1. Plot of Coarse (1a) and Fine (1b) Timing Metric Functions

point $\widehat{\eta}_{\text {center }}=\widehat{\eta}_{\text {init }}+\frac{N}{2}$. Since the length of $\boldsymbol{A}$ or $\boldsymbol{B}$ part is greater than the maximum delay spread of the multipath signal, all the four parts of the training symbol are exposed to similar multipath channel environment. A search distance of $\left[-N_{\text {cyp }}, N_{\text {cyp }}\right]$ is covered from $\widehat{\eta}_{\text {center }}$ for finding all paths of multipath channel. The timing metric is given by

$$
M_{\text {fine }}[n]=\left|P_{\text {fine }}[n]\right|^{2}
$$

with

$P_{\text {fine }}[n]=\sum_{k=0}^{\frac{N}{4}-1} r[n-k-1] \cdot r[n+k]-\sum_{k=\frac{N}{4}}^{\frac{N}{2}-1} r[n-k-1] \cdot r[n+k]$

The negative sign for $k \in\left[\frac{N}{4}, \frac{N}{2}-1\right]$ is because of the sign pattern $[+1+1+1-1], n \in\left[-N_{c y p}, N_{c y p}\right]$. This range for $n$ was chosen since the initial estimate does not produce peaks outside the maximum length of multipath channel. The timing metric produces peaks which are proportional to individual squared channel path gains. The expansion of $P_{\text {fine }}[n]$ in terms of channel coefficients is shown in Appendix. $Q[n]$ is calculated by normalizing all values of $M_{\text {fine }}[n]$ by the maximum value of $M_{\text {fine }}[n]$ such as

$$
Q[n]=\frac{M_{\text {fine }}[n]}{\max \left(M_{\text {fine }}[n]\right)}
$$

Figure $1 \mathrm{~b}$ shows the plot of $Q[n]$ for SNR of $20 \mathrm{~dB}$. The figure shows peaks corresponding to multipath gains. The threshold shown in $1 \mathrm{~b}$ helps in selecting signal only components which are to be used for the windowed summation method to find the first arrival path. The time index of the maximum value of $Q[n]$

$$
\widehat{\eta}_{\text {fine }}=\arg \max _{n}(Q[n])
$$

is used as the starting point for windowing summation method similar to Choi's. The values of $Q[n]$ are thresholded by a value $\beta$.

$$
Q[n]= \begin{cases}Q[n], & Q[n]>\beta \\ 0, & \text { otherwise }\end{cases}
$$

$\beta$ is the threshold which separates signal and noise components in $Q[n]$. This threshold is determined using the probability distribution of the noise component in $Q[n]$. The steps are as follows:

- The sequence $Q[n]$ is passed through Lloyd-Max [10] quantization algorithm using three levels of quantization.

- The lowest quantization level and its cluster is considered as noise here. It is observed that this follows a lognormal distribution. Mean $\left(\mu_{n}\right)$ and variance $\left(\sigma_{n}^{2}\right)$ of noise cluster are calculated first. The corresponding mean $\mu$ and $\sigma$ for lognormal distribution is

$$
\begin{gathered}
\mu=\log \left(\frac{\mu_{n}^{2}}{\sqrt{\sigma_{n}^{2}+\mu_{n}^{2}}}\right) \\
\sigma=\sqrt{\log \left(\frac{\sigma_{n}^{2}}{\mu_{n}^{2}}+1\right)}
\end{gathered}
$$

- A constant false alarm rate (CFAR) of $\alpha$ is used for calculation of threshold. The equation for threshold is derived by integrating probability distribution function (pdf) with limits $[\beta, \infty]$.

$$
\beta=e^{\left(\sqrt{2} \cdot \sigma \cdot e r f^{-1}(1-2 \cdot \alpha)+\mu\right)}
$$

A constant false alarm rate is maintained across all SNR values. A windowed summation is performed after discarding the noise values using the threshold $(\beta)$ calculated.

$$
E_{p}(n)=\sum_{k=0}^{S_{w}-1} Q\left(\widehat{\eta}_{\text {fine }}-n+k\right)
$$

where $S_{w}$ is the length of summation window and $J_{m}$ is the search window for signal component. Then the first arrival path is given by

$$
\widehat{\eta}_{\text {first }}=\arg \max _{n} E_{p}(n): n=0,1, \cdots J_{m}
$$

Finally,

$$
\widehat{\eta}_{\text {final }}=\widehat{\eta}_{\text {init }}-\widehat{\eta}_{\text {first }}
$$

This value indicates final estimate of the starting index of the OFDM symbol.

\section{FREQUENCY SYNCHRONIZATION}

Using estimated $\widehat{\eta}_{\text {final }}$ as the start of the training symbol, the negative sign in the training symbol is inverted to get $[\boldsymbol{C} C \boldsymbol{C} C]$. There exists some interference due to minus sign in the channel response. The frequency offset estimate is calculated using the formula

$$
\widehat{\epsilon}=\frac{2 * \angle\left(P\left[\eta_{\text {final }}\right]\right)}{\pi}
$$

where $P\left[\eta_{\text {final }}\right]$ is the autocorrelation among the four parts of the training symbol. The calculation of $P\left[\eta_{\text {final }}\right]$ is same as in $(7 \mathrm{a})$, difference being sign pattern of $[+1+1+1+1]$.

It gives both fractional and some integer frequency estimation range. The integer frequency offset estimation range depends on $L / 2$. The frequency offset estimation range of $\widehat{\epsilon}$ for proposed TS is \pm 2 sub-carrier spacing. 


\section{Simulation Results}

\section{A. Parameters}

The performance of all synchronization algorithms has been investigated by using intensive Monte-Carlo simulations $\left(10^{5}\right.$ runs). Algorithms using auto and cross-correlation techniques are compared here. The OFDM system parameters are shown in Table I. The channel used here is a frequency selective channel (ISI channel) with an exponential Power Delay Profile (PDP) and ratio of first to last Rayleigh fading tap is set to $20 \mathrm{~dB}$. A uniformly distributed random phase component is multiplied to every path during each simulation run. The channel has 16 taps with equal tap spacing of four samples. The windowing parameters used for Choi's synchronization method are $J=41, S=48$.

TABLE I

SiMULATION PARAMETERS

\begin{tabular}{|c|c|}
\hline Parameters & Value \\
\hline \hline IFFT/FFT Size $(N)$ & 1024 \\
\hline Number of sub-carriers & 1024 \\
\hline Length of Cyclic Prefix $\left(N_{c y p}\right)$ & 102 \\
\hline OFDM Symbol Length $\left(N_{s y m}\right)$ & 1126 \\
\hline Window Size $S_{w}($ samples $)$ & 40 \\
\hline Distance $J_{m}$ (samples) & 36 \\
\hline Constant False alarm rate $(\alpha)$ & 0.01 \\
\hline Number of simulation runs & $10^{5}$ \\
\hline Number of channel taps & 16 \\
\hline Channel Tap Spacing $($ samples) & 4 \\
\hline Ratio between first tap to last tap $($ in $\mathrm{dB})$ & 20 \\
\hline Carrier Frequency Offset $(\mathrm{CFO})(\epsilon)$ & 0.75 \\
\hline
\end{tabular}

\section{B. Results}

1) Timing Estimate MSE: Figure 2 shows MSE of timing estimation in the ISI channel for various synchronization methods. The delay correlation based methods (Schmidl, Minn, Shi) have higher MSE compared to methods using only conjugate symmetry correlation (Park, Choi). Park's and Choi's methods estimate maximum of the received multipath channel using conjugate symmetry correlation method. But only conjugate symmetry correlation does not give low MSE. Choi uses a windowed summation method for identifying first path, which reduces the MSE compared to Park's method. Proposed method uses conjugate symmetry and windowed summation similar to Choi to get low MSE in estimation which is better than Park and comparable to Choi at significantly lower computational complexity as shown in Figure 2.

2) Frequency Estimate MSE: Figure 3 shows the MSE of frequency estimation over a range of SNR values. A CFO of 0.75 was used during simulation. No frequency estimation was proposed for Choi and Park's methods. The Cramér-Rao bound for variance in estimation of frequency offset [7] is given by

$$
C R B(\widehat{\epsilon})=\frac{1}{2 \pi^{2}} \frac{3(S N R)^{-1}}{N\left(1-1 / N^{2}\right)}
$$

where $N$ is the size of FFT. Schmidl's frequency estimator comes closest to lower bound at all SNR values. Minn's

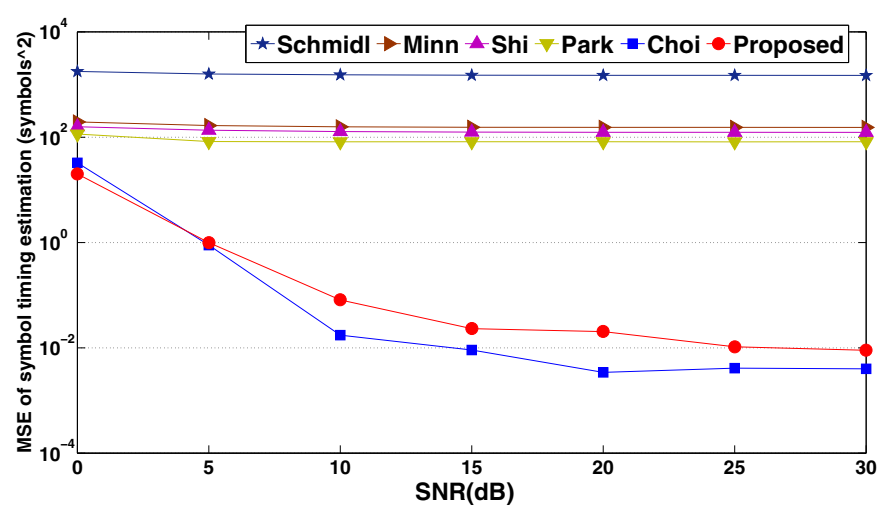

Fig. 2. MSE of Timing Estimation versus SNR in ISI channel

estimator uses algorithm of Morelli [7], which is computationally more complex compared to Schmidl's. Shi's frequency estimation algorithm hits a floor after SNR of $15 \mathrm{~dB}$. Proposed frequency estimator is similar to Schmidl's and gives estimates very close to Schmidl's at medium to high SNR values because of more accurate estimation of the starting index compared to Schmidl's algorithm which uses the same estimation formula for frequency estimation but does not suffer from interference from any negative sign in training symbol. The frequency estimator has a range of \pm 2 sub-carrier spacing compared to Schmidl's which has a range of \pm 1 sub-carrier spacing.

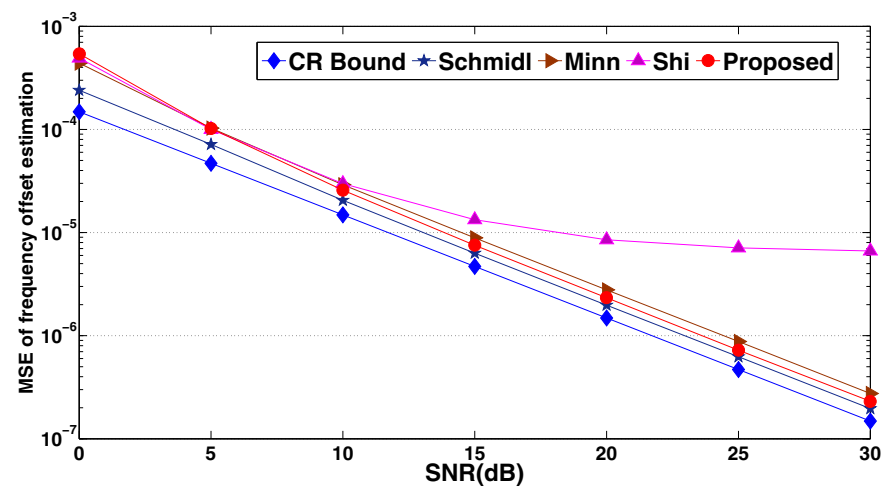

Fig. 3. MSE of Frequency Estimation versus SNR in ISI channel

3) Complexity of Calculations: The number of real operations required per symbol is shown in Table II, where $N_{\text {sym }}=N+N_{\text {cyp }}$, total OFDM symbol length. Since the algorithms of Schmidl, Minn and Shi can be written in iterative form, the number of operations is fixed per point after the initial calculation. But for algorithms of Park, Choi and Zhou where the numerator part cannot be written in the form of iterative formula, the number of operations becomes a function of size of FFT used. Since the proposed algorithm has initial iterative part and fine non-iterative part, it is also function of $N$. The non-iterative part only operates on length of $\left(2 N_{\text {cyp }}+1\right)$ and is not used on every OFDM symbol.

Comparing the proposed method with Choi, Park and Zhou's method, a saving of $\frac{N_{s y m}-\left(2 N_{c y p}+1\right)}{N_{s y m}}$ is obtained for 
TABLE II

NUMBER OF OPERATIONS PER OFDM SYMBOL

\begin{tabular}{|c|c|c|c|}
\hline Algorithm & $\begin{array}{c}\text { Real } \\
\text { Multiplication }\end{array}$ & $\begin{array}{c}\text { Real } \\
\text { Addition }\end{array}$ & Division \\
\hline \hline Schmidl-Cox & $15 N_{\text {sym }}$ & $13 N_{\text {sym }}$ & $N_{\text {sym }}$ \\
\hline Minn $(L=4)$ & $31 N_{\text {sym }}$ & $29 N_{\text {sym }}$ & $N_{\text {sym }}$ \\
\hline Shi & $59 N_{\text {sym }}$ & $61 N_{\text {sym }}$ & $N_{\text {sym }}$ \\
\hline Park & $(2 N+11) N_{\text {sym }}$ & $(2 N+7) N_{\text {sym }}$ & $N_{\text {sym }}$ \\
\hline Choi & $(2 N+7) N_{\text {sym }}$ & $(2 N+3) N_{\text {sym }}$ & $N_{\text {sym }}$ \\
\hline Zhou & $(2 N+22) N_{\text {sym }}$ & $(2 N+16) N_{\text {sym }}$ & $N_{\text {sym }}$ \\
\hline $\begin{array}{c}\text { Proposed coarse } \\
\text { step }(L=4)\end{array}$ & $31 N_{\text {sym }}$ & $29 N_{\text {sym }}$ & $N_{\text {sym }}$ \\
\hline \hline Proposed & $(2 N+3)$ & $(2 N-1)$ & $\left(2 N_{\text {cyp }}\right.$ \\
fine step & $\left(2 N_{\text {cyp }}+1\right)$ & $\left(2 N_{\text {cyp }}+1\right)$ & $+1)$ \\
\hline
\end{tabular}

Real multiplications and addition operations. This results in approximately $80 \%$ reduction in computations using numerical values of simulation compared to Choi, Park and Zhou, while MSE performance is very close to Choi's MSE.

In case of Minn, to perform the fine timing estimation, Maximum Likelihood (ML) channel estimation is done first which requires too much complexity. In case of Schmidl, the MSE is very high although it is computationally efficient. So, in terms of computational complexity, the proposed algorithm is significantly better than Choi, Park and Zhou and in terms of MSE, significantly better than Schmidl, Minn and Shi's methods. Proposed method is specially useful for MIMO systems where each antenna calculates timing synchronization and hence it needs to be computationally and resource efficient. Thus, the proposed algorithm provides a very good trade-off between computational complexity and MSE of timing and frequency estimation in a frequency selective channel.

\section{CONCLUSION}

A novel hierarchical timing estimation method for OFDM systems was presented based on new training symbol. A general method for deciding threshold based on CFAR is proposed which is calculated using noise statistics and can be adapted for different channel profiles. The proposed method is less computationally complex compared to previous methods [6], [5] and MSE is very closer to Choi's method. Compared to hybrid method of Zhou [8], proposed method saves significant number of operations due to the hierarchical approach. The amount of computations has been reduced by $80 \%$ (i.e. 5 times) of Choi or Zhou's methods in terms of number of operations. The frequency offset estimation MSE is very close to Cramér-Rao bound for frequency estimation. Thus, it provides a very good trade-off between accuracy in estimation and computational complexity.

\section{ACKNOWLEDGEMENT}

This work was supported partly by the 100GFLEX FUI project.

\section{APPENDIX}

Appendix shows the relation between fine estimation algorithm and squared channel tap gains in a multipath environment. Equation 20 shows the fine timing estimation metric used is expressed here in terms of multipath channel coefficients. The term $W[n]$ refers to the correlation terms produced due to noise and signal components. The first term in equation 20 corresponds to peaks produced in the timing metric.

\section{REFERENCES}

[1] M. Morelli, C.-C. Kuo, and M.-O. Pun, "Synchronization techniques for orthogonal frequency division multiple access (ofdma): A tutorial review," Proceedings of the IEEE, vol. 95, no. 7, pp. 1394-1427, July 2007.

[2] T. Schmidl and D. Cox, "Robust frequency and timing synchronization for OFDM," IEEE Transactions on Communications, vol. 45, no. 12, pp. 1613-1621, December 1997.

[3] H. Minn, V. Bhargava, and K. Letaief, "A robust timing and frequency synchronization for OFDM systems," IEEE Transactions on Wireless Communications, vol. 2, no. 4, pp. 822-839, July 2003.

[4] K. Shi and E. Serpedin, "Coarse frame and carrier synchronization of OFDM systems: a new metric and comparison," IEEE Transactions on Wireless Communications, vol. 3, no. 4, pp. 1271-1284, July 2004.

[5] B. Park, H. Cheon, C. Kang, and D. Hong, "A novel timing estimation method for OFDM systems," IEEE Communications Letters, vol. 7, no. 5, pp. 239-241, May 2003.

[6] S. D. Choi, J. M. Choi, and J. H. Lee, "An initial timing offset estimation method for OFDM systems in rayleigh fading channel," in IEEE 64th Vehicular Technology Conference, September 2006, pp. 1-5.

[7] M. Morelli and U. Mengali, "An improved frequency offset estimator for OFDM applications," in Communication Theory Mini-Conference, June 1999, pp. 106-109.

[8] E. Zhou, X. Hou, Z. Zhang, and H. Kayama, "A preamble structure and synchronization method based on central-symmetric sequence for ofdm," in VTC Spring, 2008, pp. 1478-1482.

[9] Y. Liu and P. Fan, "Modified chu sequences with smaller alphabet size," Electronics Letters, vol. 40, no. 10, pp. 598-599, May 2004.

[10] S. P. Lloyd, "Least squares quantization in PCM," in IEEE Transcations on Infromation Theory, vol. 2, no. 28, 1982, pp. 129-137.

$$
\begin{aligned}
P_{\text {fine }}[n] & \left.=\sum_{k=0}^{M-1} r[n-k-1] \cdot r[n+k]=\sum_{k=0}^{M-1}\left(\sum_{m=0}^{L_{h}-1} h_{m} x\left[n-k-1-\tau_{m}\right]+w[n-k-1]\right] \sum_{m^{\prime}=0}^{L_{h}-1} h_{m^{\prime}} x\left[n+k-\tau_{m^{\prime}}\right]+w[n+k]\right) \\
& =\sum_{k=0}^{M-1} \sum_{\substack{m=0, m=m^{\prime}}}^{L_{h}-1} h_{m}^{2}\left|x\left[n-k-1-\tau_{m}\right]\right|^{2}+\sum_{k=0}^{M-1}\left(\sum_{m=0}^{L_{h}-1} \sum_{\substack{m^{\prime}=0, m^{\prime} \neq m}}^{L_{h}-1} h_{m} h_{m^{\prime}} x\left[n-k-1-\tau_{m}\right] \cdot x\left[n+k-\tau_{m^{\prime}}\right]\right)+W[n] \\
P_{\text {fine }}[n] & \approx \sum_{k=0}^{M-1} \sum_{\substack{m=0, m=m^{\prime}}}^{L_{h}-1} h_{m}^{2}\left|x\left[n-k-1-\tau_{m}\right]\right|^{2}
\end{aligned}
$$

